\title{
Radiotherapy for breast cancer, the TARGIT-A trial - Authors' reply
}

Regarding the follow-up, it is tempting to speculate that in the future a difference in local recurrence will become apparent and that the trend in lower overall mortality will disappear, but neither our data ${ }^{1}$ nor previous trial results support this speculation. Statistically, to use median follow-up on its own without taking into account the absolute number of patients is inappropriate. Biologically, the temporal distribution of local recurrence shows that the first 2-3 year period covers the peak hazard of local recurrence after surgery (see figure 2 in Cheng and colleagues' paper $\left.^{2}\right)$. Importantly, results from various clinical trials have shown that the effect of local therapy such as surgery or radiation is mainly seen in the first 5 years, with the peak of the hazard by the first 2-3 years. The lines representing local recurrence between radiotherapy and no radiotherapy in Kaplan-Meier plots remain almost parallel after 5 years in the NSABP B06, NSABP B04, and the Oxford Overview. The conclusion of the 25-year follow-up of the Swedish trial ${ }^{\frac{3}{2}}$ of radiotherapy versus no radiotherapy was explicit:

"Radiotherapy protects against recurrences during the first 5 years of follow-up." Whatever difference was going to be noted at 25 years was already seen at 5 years, with most of the difference already seen by 2-3 years (as seen figure 2A in Wickberg and colleagues' paper $^{3}$ ). The TARGIT-A trial ${ }^{l}$ has a substantial number of patients $(n=1222)$ with a median follow-up of 5 years, and 2232 patients had a median follow-up of nearly 4 years.

Regarding non-breast cancer deaths, we found that in the 52 deaths we have recorded so far, the ratio of TARGIT:EBRT was 17:35; such a notable baseline imbalance in cardiac morbidity favouring TARGIT seems unlikely in a randomised trial of this size. We noted a significant difference in non-breast cancer mortality with a high degree of confidence $(p=0 \cdot 0086)$, which seems to lead to a trend in reduced overall mortality with TARGIT. ${ }^{1}$

Our data are consistent with the recent analysis of Darby and colleagues. ${ }^{4}$ They reported that the risk is highest in the first 10 years - during this period, the risk of cardiac mortality is increased by $16.3 \%$ $(95 \%$ CI $3 \cdot 0-64 \cdot 3)$ - ie, a risk ratio of $1 \cdot 163(1 \cdot 03-1 \cdot 643)$ per Gy. ${ }^{4}$ Presence of ischaemic heart disease (equally balanced between cases and controls) has a multiplicative effect and the risk ratio rises to $13.4 \%$ (95\% CI 7.65-23.58) per Gy. As the risk increases linearly with dose, for a typical patient with an exposure of $3 \mathrm{~Gy}$, the risk would be three-times higher with a relatively wide upper confidence limit. These estimates are consistent with the TARGIT-A trial results. ${ }^{1}$

One might expect irradiation for left-sided cancers to result in higher cardiac toxic effects. However, the ratio of cardiac risk of left:right sided cancers is small $(1 \cdot 34$ as per Darby and colleagues). ${ }^{4}$ Furthermore, Darby and colleagues ${ }^{4}$ recorded no significant effect of laterality on cardiac toxic effects per Gy. With modern radiotherapy designed to reduce cardiac dose, the absolute difference between sides is likely to be even lower and undetectable with few events. So an absence of a difference between the left and right sides should not be interpreted as an absence of cardiac toxicity.

In the TARGIT-A trial, $\frac{1}{}$ the 5 -year risk of non-breast cancer mortality was $1.4 \%$ (TARGIT) versus $3.5 \%$ (EBRT). The absolute difference in non-breast-cancer mortality was $2 \cdot 1 \%$, about a third of which $(0.6 \%)$ was from cardiac causes. This small increase in non-breast cancer mortality might have been uncovered early in the TARGIT-A trial because of the otherwise excellent outcome from breast cancer (5-year mortality 2.2\%). In older trials, this small but lethal effect might have been masked until the early high breast cancer mortality (eg, $30 \%$ at 5 years 
in the CRC1 trial) diminished in later years.

Furthermore, intraoperative irradiation of a fresh tumour bed has been shown to abrogate the stimulatory and inflammatory effects of surgical wounding. ${ }^{-}$Could this possibly have systemic beneficial effects that contribute to the reduction in nonbreast-cancer mortality? This bold conjecture is supported by the observation of a significant reduction in non-breast cancer mortality when patients receive TARGIT plus EBRT compared with EBRT alone. ${ }^{-}$The hypothesis can be fortuitously tested in the TARGIT-B superiority trial in higher risk women (TARGIT boost plus EBRT vsEBRT boost plus EBRT).

Although non-inferiority trials are becoming more common, especially when cure rates are high and a reduction in treatment toxicity without a loss of efficacy is desirable, the concept of noninferiority can be difficult to grasp. The TARGIT-A trial was such a non-inferiority trial, which means that even if there is a significant difference, as long as it is less than a prespecified value $(2.5 \%$ in the TARGIT-A trial), the two groups are judged non-inferior to each other. In these circumstances, less expensive, less toxic, or more convenient treatment becomes the preferred choice.

Furthermore, we had prespecified that the significant $p$ value for difference for the log-rank test would be less than 0.01 for local recurrence-as this was the second such analysis. So the $2 \%$ difference was not only within the non-inferiority margin but also, since the $p$ value was $0 \cdot 04$, strictly speaking, it was not statistically significant.

We have used the standard method of using binomial proportions to calculate the non-inferiority statistic. Single 5-year point estimates do not represent absolute events and can lead to erroneous conclusions, especially when the event rate is low. The method we have used makes a comprehensive assessment of the whole follow-up period, which stretches from the day of randomisation to the longest follow-up of more than 12 years and includes all events in that period. To address the issue of follow-up, we repeated the analysis by restricting the cohorts randomised early in the trial including the group of 1222 patients with a median follow-up of 5 years. The multiple comparisons issue does not apply as we have not chosen one of several comparisons to make our conclusions. We have used all three cohorts together to inform the discussion and clearly stated that they are nested within each other. Exploratory analysis of regional recurrence by intention to treat showed no difference between the two randomised groups. Furthermore, omission of EBRT did not increase axillary recurrence when analysed as per treatment received: the number of axillary recurrences were five of 1613 when EBRT was not given versus six of 1762 when EBRT was given; 5-year risks $0.68 \%(95 \%$ CI $0.28-1 \cdot 6)$ versus $0 \cdot 82 \%(0 \cdot 34-2 \cdot 02)$, HR $0 \cdot 84(0 \cdot 26-2 \cdot 74$, $\mathrm{p}=0 \cdot 8)$. $^{\mathrm{7}}$

Despite a clear description of how the prepathology and postpathology strata were prespecified during the course of the trial, Jack Cuzick refers to these as subgroups. He suggests that inability to find heterogeneity is a proof of its absence. The total number of recurrences is low, and there might not be enough power to detect heterogeneity between the two strata even though it might exist. In fact, a Cox proportional hazards analysis showed that the timing of randomisation variable was significant. Furthermore, the formal test for non-inferiority showed that although prepathology TARGIT was non-inferior, postpathology was not. We have been cautious in our discussion. 1

In response to Jay Harness and colleagues, we believe that calculaton of the dose to the heart in only those who died of myocardial infarction is not meaningful when its proportional attribution to the death is small. Additionally, no patient 
who was randomly assigned to EBRT but received TARGIT died of a myocardial infarction. We did not record detailed cardiovascular assessment and excluded only those patients with severe comorbidities. Finally, we included deaths from stroke and ischaemic bowel under cardiovascular, not cardiac, events.

We believe that clinicians and health-care policy makers should not deny suitable

\section{References}

1. Vaidya, JS, Wenz, F, Bulsara, M..., and on behalf of the TARGIT trialists' group. Risk-adapted targeted intraoperative radiotherapy versus whole-breast radiotherapy for breast cancer: 5-year results for local control and overall survival from the TARGIT-A randomised trial. Lancet. 2014; 383:603-613

2. Cheng, L, Swartz, MD, Zhao, $\mathrm{H}$ et al. Hazard of recurrence among women after primary breast cancer treatment - a 10 year follow up using data from SEERMedicare. Cancer Epidemiol Biomarkers Prev. 2012; 21: 800-809

3. Wickberg, A, Holmberg, L, Adami, HO, Magnuson, A, Villman, K, and Liljegren, G. Sector resection with or without postoperative radiotherapy for stage I breast cancer: 20-year results of a randomized trial. J Clin Oncol. 2014; 32: 791-797

4. Darby, SC, Ewertz, M, McGale, P et al. Risk of ischemic heart disease in women after radiotherapy for breast cancer. $N$ Engl J Med. 2013; 368: 987-998

5. Belletti, B, Vaidya, JS, D'Andrea, S et al. Targeted intraoperative radiotherapy impairs the stimulation of breast cancer cell proliferation and invasion caused by surgical wounding. Clin Cancer Res. 2008; 14: 1325-1332

6. Vaidya, JS, Bulsara, M, Wenz, F et al. The lower non-breast cancer mortality with TARGIT in the TARGIT-A trial could be a systemic effect of TARGIT on tumor microenvironment. Int J Radiat Oncol Biol Phys. 2013; 87: S240

7. Vaidya, JS, Wenz, F, Bulsara, M et al. Omitting whole breast radiation therapy did not increase axillary recurrence in the TARGIT-A trial. Int J Radiat Oncol Biol Phys. 2013; 87: S7 